\title{
Powering Multiparameter Homotopy-Based Simulation with a Fast Path-Following Technique
}

\author{
Héctor Vázquez-Leal, ${ }^{1}$ Roberto Castañeda-Sheissa, ${ }^{1}$ \\ Felipe Rabago-Bernal, ${ }^{2}$ Luis Hernández-Martínez, ${ }^{3}$ \\ Arturo Sarmiento-Reyes, ${ }^{3}$ and Uriel Filobello-Niño ${ }^{1}$ \\ ${ }^{1}$ Electronic Instrumentation and Atmospheric Sciences School, University of Veracruz, \\ 91000 Xalapa, VER, Mexico \\ ${ }^{2}$ Institute of Physics, Autonomous University of San Luis Potosi, 78000 San Luis Potosi, SLP, Mexico \\ ${ }^{3}$ Electronics Department, National Institute for Astrophysics, Optics and Electronics, \\ 72000 Tonantzintla, PUE, Mexico
}

Correspondence should be addressed to Héctor Vázquez-Leal, hvazquez@uv.mx

Received 30 June 2011; Accepted 23 August 2011

Academic Editors: F. Jauberteau and C. I. Siettos

Copyright (C) 2011 Héctor Vázquez-Leal et al. This is an open access article distributed under the Creative Commons Attribution License, which permits unrestricted use, distribution, and reproduction in any medium, provided the original work is properly cited.

The continuous scaling for fabrication technologies of electronic circuits demands the design of new and improved simulation techniques for integrated circuits. Therefore, this work shows how the hypersphere technique can be adapted and applied to trace a multiparameter homotopy. Besides, we present a path-following technique based on circles (evolved from hypersphere), which is faster, and simpler to be implemented than hypersphere technique. Last, a comparative analysis between both techniques applied to simulation of circuits with bipolar transistors will be shown.

\section{Introduction}

The increment of the complexity of circuits influence the scientific progress in the simulation techniques area for integrated circuits. Also, homotopy techniques have been introduced as a useful tool in the area of operating point solution for circuits [1-5], due to the NewtonRaphson (NR) method (widely used) which shows convergence problems [6] like oscillation and divergence.

\section{Multiparameter Homotopy}

The first step to formulate a homotopy is to establish the equilibrium equation to be solved; it is formulated from Kirchhoff laws, being defined as

$$
f(x)=0 \text {, where } f: \in \mathfrak{R}^{n} \longrightarrow \mathfrak{R}^{n},
$$


where $x$ represents the electrical variables of the circuit and $n$ is the number of electrical variables.

Multiparameter homotopies [7-9] are characterized by adding more than one extra homotopy parameter to the equilibrium equation. When homotopy parameters are adjusted to zero, the solution for $H(\cdot)$ becomes trivial, and when parameters reach value of one, then the operating point is located. The multiparameter homotopy function can be represented as

$$
H\left(f(x), \lambda_{1}, \lambda_{2}, \ldots, \lambda_{k}\right)=0,
$$

where homotopy parameters are $\lambda_{1}, \lambda_{2}, \ldots, \lambda_{k} \in[0,1]$ and $k$ is the number of homotopy parameters.

Multiparameter homotopy [7] has been proposed in order to avoid fork bifurcations, singularities, among other problems that can be encountered with homotopy paths. Besides, as for the uniparametric [2] and multiparameter homotopies, the tracing technique $[10,11]$ is a fundamental tool capable of affecting the convergence, speed, and number of solutions located. Therefore, it is proposed to apply two tracing techniques for multiparameter homotopy, both will be described in the following sections.

\section{Tracing Techniques}

In order to apply tracing techniques described in this paper, a biparametric homotopy based in Newton's homotopy method will be used as an example:

$$
H\left(f(x), \lambda_{1}, \lambda_{2}\right)=f\left(x, \lambda_{2}\right)-\left(1-\lambda_{1}\right) f\left(x_{i}, 0\right), \quad \text { where } H: \in \mathfrak{R}^{n+1} \times \mathfrak{R} \longrightarrow \mathfrak{R}^{n}
$$

With the existence of two parameters $\left(\lambda_{1}\right.$ and $\left.\lambda_{2}\right)$, two simultaneous deformations or transformations are produced: one in function $f$ and another in function $H$. When $\left[x, \lambda_{1}, \lambda_{2}\right]=$ $\left[x_{i}, 0,0\right]$, then

$$
H\left(f(x), \lambda_{1}, \lambda_{2}\right)=f\left(x_{i}, 0\right)-f\left(x_{i}, 0\right)=0 .
$$

Hence, homotopy function is satisfied. Besides, when $\left[\lambda_{1}, \lambda_{2}\right]=[1,1]$ becomes

$$
H\left(f(x), \lambda_{1}, \lambda_{2}\right)=f(x)
$$

so the found solution of $H$ is the solution of the equilibrium equation. Nonetheless, as function $H$ has two extra variables, it is necessary to add two equations to the system $H$ in order to be solved using more conventional techniques like NR. 


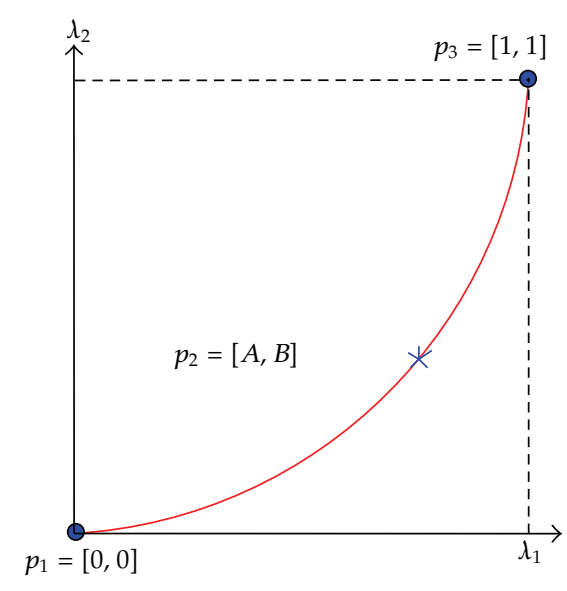

(a)

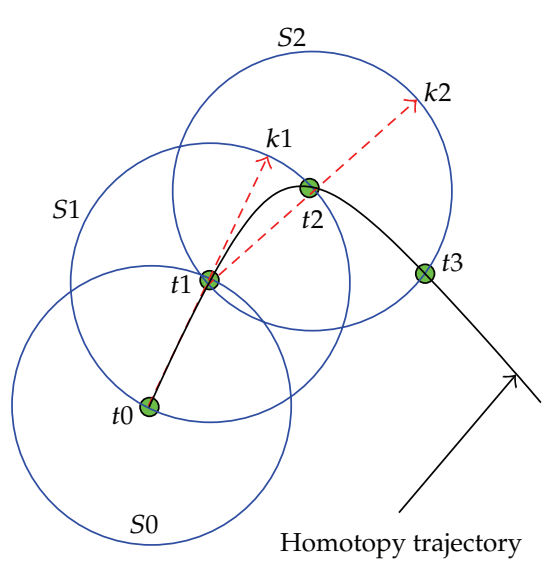

(b)

Figure 1: (a) Parametric function. (b) Hypersphere technique.

\section{(1) Equation $n+1$}

One equation is added to define path $\lambda_{1}-\lambda_{2}$, which will be named parametric function $M\left(\lambda_{1}, \lambda_{2}\right)$. This equation traverses three points $\left[\lambda_{1}, \lambda_{2}\right]: p_{1}=[0,0], p_{2}=[A, B]$, and $p_{3}=[1,1]$. The proposed equation is

$$
M\left(\lambda_{1}, \lambda_{2}\right)=-\lambda_{1}+\frac{\left(\lambda_{2}+(B(-1+A) /(A B+1-2 A))\right)}{\left(-\left((-1+2 A-B) \lambda_{2} /(A B+1-2 A)\right)+2(B(-1+A) /(A B+1-2 A))\right)},
$$

where $p_{2}$ is defined by user, as shown in Figure $1(\mathrm{a})$. The range of values for $A$ and $B$ is $[0,1]$.

\section{(2) Equation $n+2$}

Hypersphere equation is added [12]:

$$
S(\cdot)=\left(x_{1}-c_{1}\right)^{2}+\left(x_{2}-c_{2}\right)^{2}+\cdots+\left(\lambda_{1}-c_{n+1}\right)^{2}+\left(\lambda_{2}-c_{n+2}\right)^{2}-r^{2},
$$

where $c$ is the center of the hypersphere (which adjusts its value each iteration) and $r \ll 1$ is the hypersphere radius (step size).

The summary of the procedures consists in the following steps [12] (see Figure 1(b)).

(1) The first sphere is established $S 0$ with center located at $t 0=\left[x_{i}, p_{1}\right]$ and the equation system is solved (3.1), (3.4), and (3.5) using the NR method (setting t0 as an initial point), locating point $t 1$.

(2) A new hypersphere $S 1$ is created with center at $t 1$.

(3) Using points $t 0$ and $t 1$, it is possible to create a prediction, which touches hypersphere $S 1$ at point $k_{1}$; it is used as initial point for the NR method, until locating point $t_{2}$ on the homotopy path. 
(4) Steps 2 and 3 are successively repeated (updating hypersphere's center after each iteration) until crossing point $p_{3}$.

(5) Points before and after $p_{3}$ are used to perform an interpolation [13]. The type of interpolation employed in this paper is linear multidimensional interpolation, which produces an approximation $x_{a}$ of solution $x_{s}$ for the equilibrium equation.

(6) Finally, using as initial point $x_{a}$ in the NR method, the precision for the operating point $x_{s}$ is improved.

It is possible to replace (3.5) for the circle equation, in function of the homotopy parameters:

$$
C(\cdot)=\left(\lambda_{1}-c_{n+1}\right)^{2}+\left(\lambda_{2}-c_{n+2}\right)^{2}-r^{2}
$$

where $r \ll 1$. The rest of the steps to implement the numerical continuation are the same as the hypersphere technique already described.

\section{Study Case: Circuit with Bipolar Transistors and a Diode}

The following circuit [14] (see Figure 2) contains nine solutions and has become the reference circuit for the homotopy applied to circuit analysis. Using the system reported by [14], equilibrium equation is augmented

$\mathbf{f}\left(v_{1}, v_{2}, v_{3}, v_{4}, \lambda_{2}\right)=\left\{\begin{aligned} f_{1}= & 6.103168 I_{S}\left(e^{40 v_{1}}-1\right) \lambda_{2}+4.36634 v_{2}+2.863168 I_{S}\left(e^{40 v_{2}}-1\right)-12, \\ f_{2}= & 5.4 v_{1}+3.58 I_{S}\left(e^{40 v_{1}}-1\right) \lambda_{2}+6.62 I_{S}\left(e^{40 v_{2}}-1\right)+v_{3} \\ & +0.7 I_{S}\left(e^{40 v_{3}}-1\right)+0.5 I_{S}\left(e^{40 v_{4}}-1\right)-22 \\ f_{3}= & 6.103168 I_{S}\left(e^{40 v_{3}}-1\right)+2.863168 I_{S}\left(e^{40 v_{4}}-1\right) \lambda_{2}+4.36634 v_{4}-12, \\ f_{4}= & v_{1}+0.7 I_{S}\left(e^{40 v_{1}}-1\right) \lambda_{2}+0.5 I_{S}\left(e^{40 v_{2}}-1\right)+5.4 v_{3} \\ & +3.58 I_{S}\left(e^{40 v_{3}}-1\right)+6.62 I_{S}\left(e^{40 v_{4}}-1\right) \lambda_{2}-20,\end{aligned}\right.$

where $I_{s}=10^{-6},\left[v_{1}, v_{2}, v_{3}, v_{4}\right]$ are the voltage drop between base-emitter terminals for each transistor in the circuit (see Figure 2) and $\lambda_{2}$ is the second homotopy parameter. The complete homotopy formulation can be established by using the following equations:

(i) the augmented equilibrium equation (4.1),

(ii) the homotopy function equation (3.1),

(iii) the parametric function (3.4),

(iv) the hypersphere function (3.5) or circle function (3.6), which depends on the selected technique.

Table 1 presents in summary the results of performing the tracing of four paths with different initial points $\left(x_{i 1}, x_{i 2}, x_{i 3}\right.$, and $\left.x_{i 4}\right)$, each one. This process was repeated for both tracing techniques, showing the results in Figures 3(a) and 3(b). Comparing both techniques, 


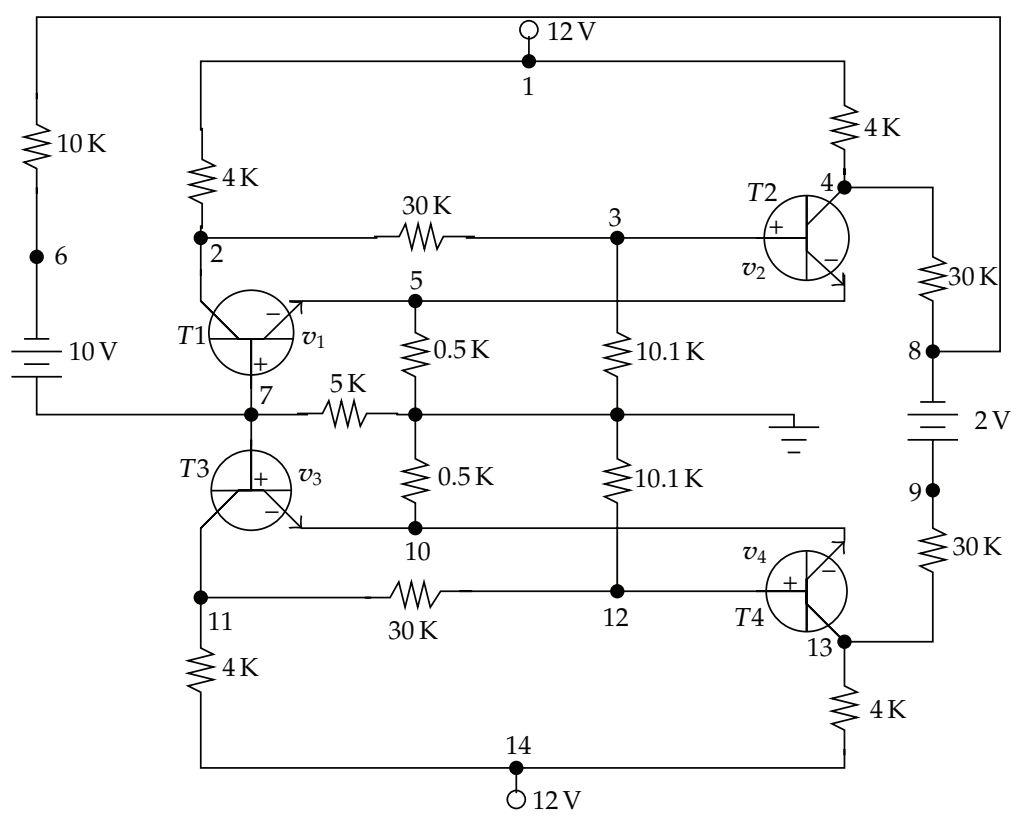

Figure 2: Chua's circuit.

Table 1: Relevant points for homotopy simulations.

\begin{tabular}{|c|c|c|c|}
\hline $\begin{array}{l}\text { Hypersphere-Init. point } \\
\text { where }\left[\lambda_{1}, \lambda_{2}\right]=[0,0]\end{array}$ & No. Iter & Time (Sec) & $\begin{array}{c}\text { Operating point }\left[v_{1}, v_{2}, v_{3}, v_{4}\right] \\
\text { where }\left[\lambda_{1}, \lambda_{2}\right]=[1,1]\end{array}$ \\
\hline$x_{i 1}=[-5,-5,-5,-5]$ & 519 & 7.70 & $x_{s 1}=[0.3830,-3.5446,0.3851,-4.0990]$ \\
\hline$x_{i 2}=[-1,-2,-1,0]$ & 202 & 3.59 & $x_{s 2}=[0.3869,-4.6321,-0.8002,0.3775]$ \\
\hline$x_{i 3}=[-5,-0.5,-5,0]$ & 216 & 4.06 & $x_{s 3}=[-0.5136,0.3775,-0.9682,0.3775]$ \\
\hline$x_{i 4}=[-1,0,0,0]$ & 168 & 3.13 & $x_{s 4}=[-1.0510,0.3775,0.3845,-3.9542]$ \\
\hline $\begin{array}{l}\text { Circle-Init. point } \\
\text { where }\left[\lambda_{1}, \lambda_{2}\right]=[0,0]\end{array}$ & No. Iter & Time (Sec) & $\begin{array}{c}\text { Operating point }\left[v_{1}, v_{2}, v_{3}, v_{4}\right] \\
\text { where }\left[\lambda_{1}, \lambda_{2}\right]=[1,1]\end{array}$ \\
\hline$x_{i 1}=[-5,-5,-5,-5]$ & 48 & 0.86 & $x_{s 1}=[0.3830,-3.5446,0.3851,-4.0990]$ \\
\hline$x_{i 2}=[-1,-2,-1,0]$ & 48 & 0.89 & $x_{s 2}=[0.3869,-4.6321,-0.8002,0.3775]$ \\
\hline$x_{i 3}=[-5,-0.5,-5,0]$ & 48 & 0.74 & $x_{s 4}=[-0.5136,0.3775,-0.9682,0.3775]$ \\
\hline$x_{i 4}=[-1,0,0,0]$ & 48 & 0.82 & $x_{s 4}=[-1.0510,0.3775,0.3845,-3.9542]$ \\
\hline
\end{tabular}

there are two interesting conclusions to be highlighted: first, homotopy paths traced from the same initial point lead to the same solution; in fact, comparing paths point by point, it can be observed that it is the same path; second, despite that paths are identical, the circle technique required a fixed number of iterations (48); those are much less required than using the hypersphere method. In fact, from Table 1, it can be concluded that, at best (choosing initial point at $x_{i 1}$ ), the tracing technique for circles required 8.95 times less CPU time (by using MAPLE 15 software in an Intel Quad Core i7 processor at $2.6 \mathrm{GHz}$ ) than the hypersphere technique. Both tracing techniques employed radius of $r=0.03$ and a parametric function $M$ with $p_{2}=[0.2,0.3]$. 


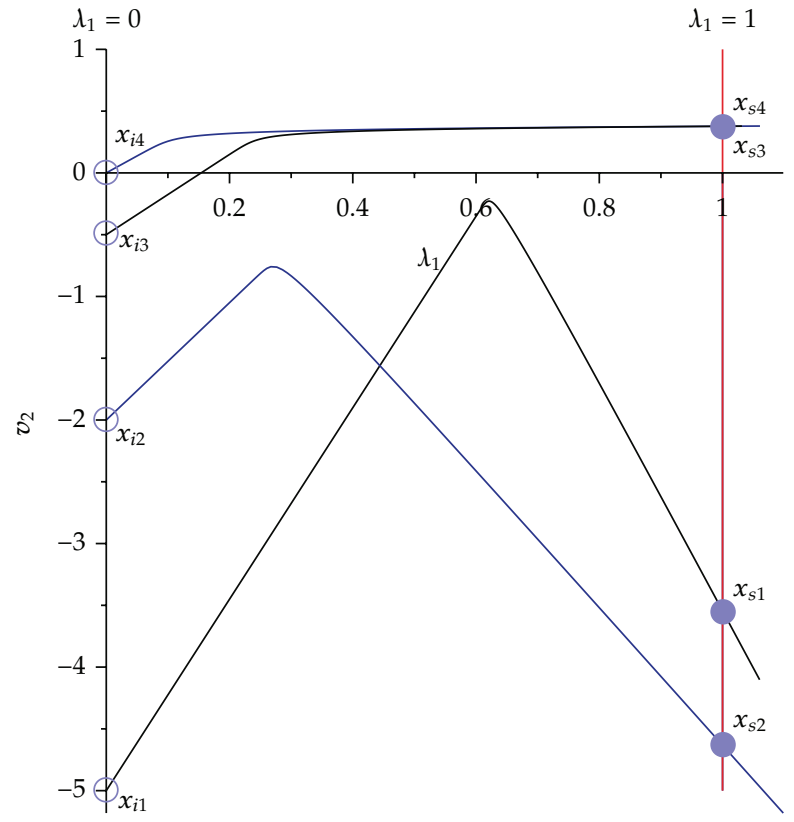

(a) Hypersphere technique

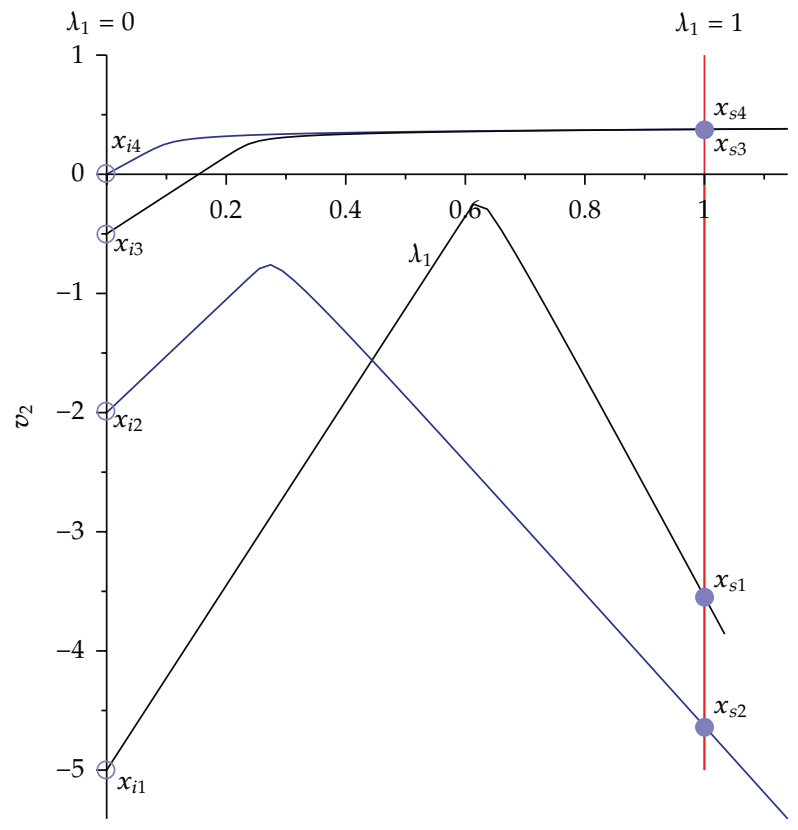

(b) Circle technique

Figure 3: Homotopy trajectories $v_{2}-\lambda_{1}$.

Circles technique can be modified changing one of the homotopy parameters by an electrical variable of interest. For instance, the simulation was repeated from initial point $x_{i 1}$, only changing the circle from (3.6) by

$$
C\left(v_{1}, \lambda_{1}\right)=v_{1}^{2}+\lambda_{1}^{2}-r^{2},
$$


where $v_{1}, r \in \Re$. The result was that the homotopy path already known was traced (see Figure 3(b)) with a total of 191 iterations (locating the same solution at $x_{s 1}$ ). Also, it is possible to use one of the two homotopy parameters with more than one electrical variable, to implement a reduced hypersphere. Therefore, in a forthcoming work the study of circles technique will be expanded and a possible application to simulate VLSI circuits will also be discussed.

\section{Conclusion}

This work showed that it is possible to use the hypersphere technique to trace multiparameter homotopies. Besides, a tracing technique derived from hypersphere (circles) was introduced, which is simpler to program and faster than the hypersphere technique. These results make the circles technique an attractive tool to trace multiparameter homotopies.

\section{References}

[1] L. B. Goldgeisser and M. M. Green, "A method for automatically finding multiple operating points in nonlinear circuits," IEEE Transactions on Circuits and Systems-I, vol. 52, no. 4, pp. 776-784, 2005.

[2] R. C. Melville and L. Trajković, "Artificial parameter homotopy methods for the DC operating point problem," IEEE Transactions on Computer-Aided Design of Integrated Circuits and Systems, vol. 12, no. 6, pp. 861-877, 1997.

[3] L. Trajković, R. C. Melville, and S. C. Fang, "Passivity and no-gain properties establish global convergence of a homotopy method for DC operating points," in Proceedings of the IEEE International Symposium on Circuits and Systems, 1990.

[4] H. Vázquez-Leal, L. Hernández-Martínez, and A. Sarmiento-Reyes, "Double-bounded homotopy for analysing nonlinear resistive circuits," in Proceedings of the IEEE International Symposium on Circuits and Systems, May 2005.

[5] J. S. Roychowdhury and R. C. Melville, "Homotopy techniques for obtaining a DC solution of largescale MOS circuits," in Proceedings of the 33rd Annual Design Automation Conference, 1996.

[6] C. T. Kelley, Solving Nonlinear Equations with Newton's Method, Fundamentals of Algorithms, SIAM, Philadelphia, Pa, USA, 2003.

[7] D. M. Wolf and S. R. Sanders, "Multiparameter homotopy methods for finding DC operating points of nonlinear circuits," IEEE Transactions on Circuits and Systems-I, vol. 43, no. 10, pp. 824-838, 1996.

[8] J. Roychowdhury and R. Melville, "Delivering global DC convergence for large mixed-signal circuits via homotopy/continuation methods," IEEE Transactions on Computer-Aided Design of Integrated Circuits and Systems, vol. 25, no. 1, pp. 66-78, 2006.

[9] H. Vázquez-Leal, L. Hernández-Martínez, A. Sarmiento-Reyes, and R. S. Murphy-Arteaga, "Improving multi-parameter homotopy via symbolic analysis techniques for circuit simulation," in Proceedings of the European Conference on Circuit Theory and Design, vol. 2, pp. 402-405, 2003.

[10] H. Vázquez-Leal, L. Hernández-Martínez, and A. Sarmiento-Reyes, "Numerical continuation scheme for tracing the double bounded homotopy for analysing nonlinear circuits," in Proceedings of the International Conference on Communications, Circuits and Systems, 2005.

[11] E. L. Allgower and K. Georg, Numerical Path Following, 1994.

[12] K. Yamamura, "Simple algorithms for tracing solution curves," IEEE Transactions on Circuits and Systems I, vol. 40, no. 8, pp. 537-541, 1993.

[13] M. Sosonkina, L. T. Watson, and D. E. Stewart, "Note on the end game in homotopy zero curve tracking," ACM Transactions on Mathematical Software, vol. 22, no. 3, pp. 281-287, 1996.

[14] A. Ushida and L. O. Chua, "Tracing solution curves of nonlinear equations with sharp turning points," International Journal of Circuit Theory and Applications, vol. 12, no. 1, pp. 1-21, 1984. 


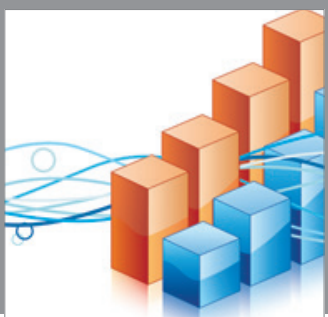

Advances in

Operations Research

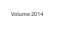

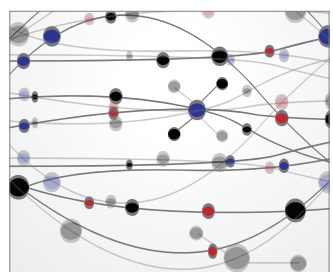

\section{The Scientific} World Journal
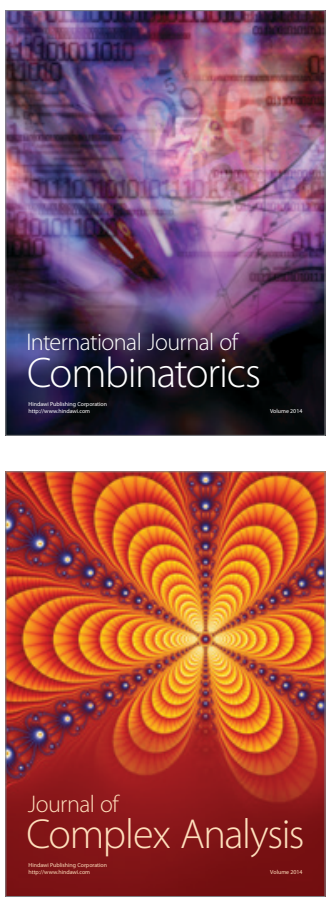

International Journal of

Mathematics and

Mathematical

Sciences
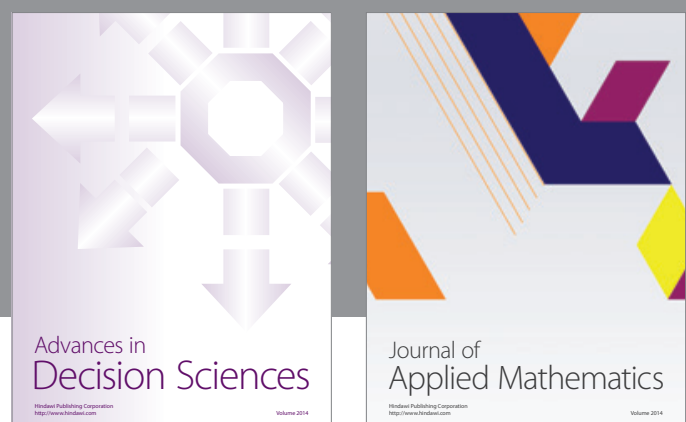

Journal of

Applied Mathematics
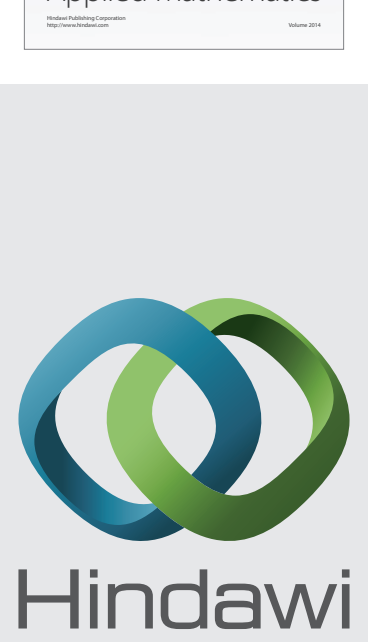

Submit your manuscripts at http://www.hindawi.com
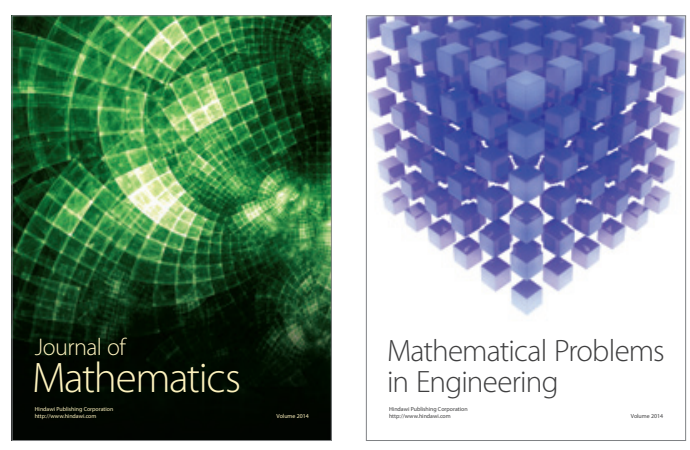

Mathematical Problems in Engineering
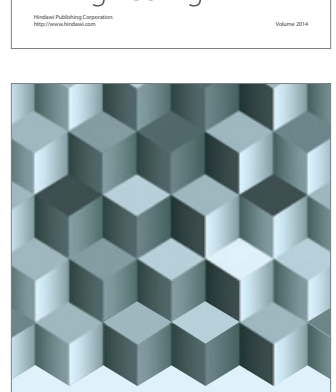

Journal of

Function Spaces
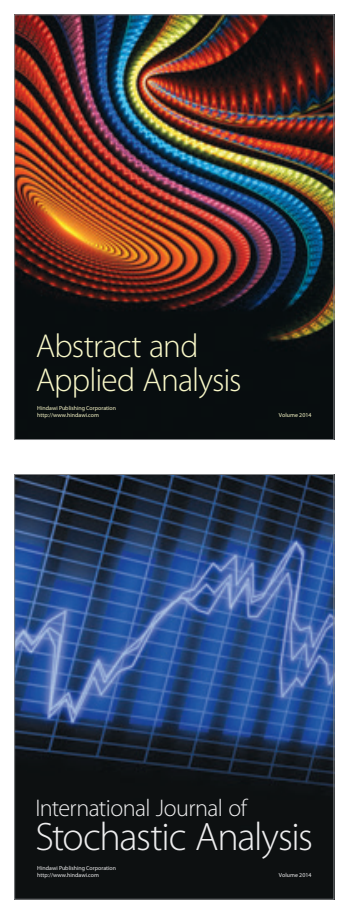

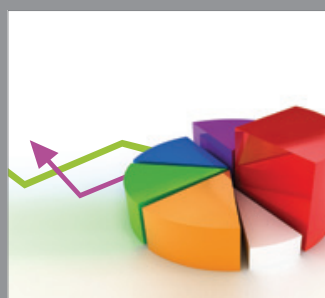

ournal of

Probability and Statistics

Promensencen
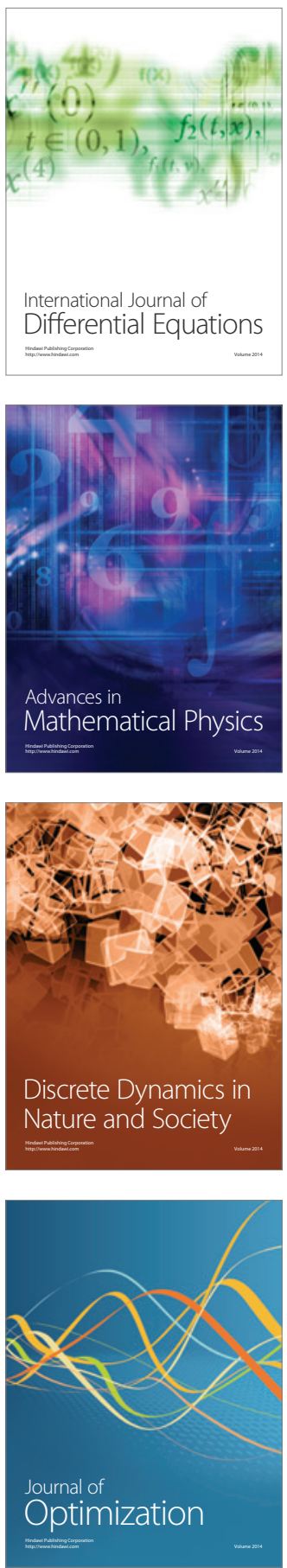